\title{
Serial Changes in Clinical Presentations and Outcomes of 5,740 Patients Requiring Repeated Hospital Admissions (Four or More Times) due to Worsened Heart Failure
}

\author{
Hidetaka Itoh, ${ }^{1} \mathrm{MD}$, Hidehiro Kaneko, ${ }^{1,2} \mathrm{MD}$, Haruki Yotsumoto, ${ }^{1} \mathrm{BS}$, Hiroyuki Kiriyama, ${ }^{1} \mathrm{MD}$, \\ Tatsuya Kamon, ${ }^{1} \mathrm{MD}$, Katsuhito Fujiu, ${ }^{1,2} \mathrm{MD}$, Kojiro Morita, ${ }^{3,4} \mathrm{PhD}$, Nobuaki Michihata, ${ }^{5} \mathrm{MD}$, \\ Taisuke Jo, ${ }^{5}$ MD, Norifumi Takeda, ${ }^{1}$ MD, Hiroyuki Morita, ${ }^{1}$ MD, \\ Hideo Yasunaga, ${ }^{3} \mathrm{MD}$ and Issei Komuro, ${ }^{1} \mathrm{MD}$
}

\begin{abstract}
Summary
Heart failure (HF) is a major cause of death and hospitalization worldwide. In particular, hospital readmission due to worsened HF occurs frequently after the onset of HF. However, the association of repeated hospital admission with clinical manifestations and outcomes is unclear. The aim of this study was to clarify the serial changes in presentation and clinical course of patients requiring repeated hospital admission due to worsened HF. Among 466,921 patients who were admitted and discharged between January 2010 and March 2018, with the main discharge diagnosis of HF, we studied 5,740 patients who were hospitalized 4 times or more, using the Diagnosis Procedure Combination database. We evaluated serial changes in continuous data using the Jonckheere trend test, and categorical data using the Cochran-Armitage trend test. The median age of the patients was 78 years, and 3,326 patients (58\%) were male. Body mass index and Barthel Index decreased with increased numbers of admissions. Patients requiring respiratory support and hemodialysis increased, whereas patients undergoing intra-aortic balloon pumping decreased with increased numbers of admissions. The length of hospital stay was prolonged and the interval between hospitalizations was shortened with increased numbers of hospital admissions. The in-hospital mortality rate was $8.8 \%$ at the fourth admission. In conclusion, this is the first large-scale real-world study on the serial changes in characteristics and outcomes of HF patients requiring repeated hospitalization, suggesting that repeated hospitalization might adversely affect the general status of patients with HF and result in a vicious clinical cycle.
\end{abstract}

Key words: Acute Heart Failure, Readmission, Epidemiology

(Int Heart J 2020; 61: 1253-1257)

$\mathrm{H}$ eart failure (HF) is a major cause of death and hospital admission worldwide. ${ }^{1-3)}$ Hospital admission due to worsened HF is an important dimension of the clinical practice of patients with HF, ${ }^{4,5}$ and readmission due to worsened HF occurs frequently after the onset of HF. ${ }^{2,6)}$ Furthermore, previous studies have showed that prior history of hospital admission was associated with poor clinical outcomes for HF patients. ${ }^{7-10}$ Readmission due to worsened HF could affect the clinical presentation of patients with HF, and result in deteriorated outcomes. Although Gheorghiade, et al indicated that a further decrease in cardiac function could occur with each hospital admission for acute HF, repeated episodes of worsened HF could also lead to non-cardiac damage, such as progression of cachexia, and a decrease in the activity of daily living. However, little is known about the association of repeated admissions due to worsened HF with clinical manifestations such as body weight and activities of daily living, and in-hospital outcomes of patients with HF. In this study, we aimed to explore the serial changes in clinical presentation and outcomes of patients requiring repeated hospital admissions due to worsened HF using a nationwide inpatient database.

\section{Methods}

Data source and study design: We performed a retrospective cohort study using the Diagnosis Procedure Combination database, a nationwide inpatient database in Japan. ${ }^{11-13)}$ The database includes administrative claims data

From the ${ }^{1}$ Department of Cardiovascular Medicine, Graduate School of Medicine, The University of Tokyo, Tokyo, Japan, ${ }^{2}$ Department of Advanced Cardiology, Graduate School of Medicine, The University of Tokyo, Tokyo, Japan, ${ }^{3}$ Department of Clinical Epidemiology and Health Economics, School of Public Health, The University of Tokyo, Tokyo, Japan, ${ }^{4}$ Department of Health Services Research, Faculty of Medicine, University of Tsukuba, Ibaraki, Japan and ${ }^{5}$ Department of Health Services Research, The University of Tokyo, Tokyo, Japan.

This work was supported by grants from the Ministry of Health, Labor and Welfare, Japan (19AA2007 and H30-Policy-Designated-004) and the Ministry of Education, Culture, Sports, Science and Technology, Japan (17H04141).

Address for correspondence: Hidehiro Kaneko, MD, Department of Cardiovascular Medicine, The University of Tokyo Hospital, 7-3-1, Hongo, Bunkyo-ku, Tokyo, 113-8655, Japan. E-mail: hidehikaneko-circ@umin.ac.jp or kanekohidehiro@gmail.com

Received for publication June 19, 2020. Revised and accepted August 17, 2020.

Released in advance online on J-STAGE November 13, 2020.

doi: 10.1536/ihj.20-441

All rights reserved by the International Heart Journal Association. 
Table I. Characteristics of Study Population

\begin{tabular}{|c|c|}
\hline Variable & \\
\hline Number of patients & 5,740 \\
\hline Age (years) & $78(70-84)$ \\
\hline $20-59$ & $421(7.3)$ \\
\hline $60-69$ & $921(16.0)$ \\
\hline $70-79$ & $1,842(32.1)$ \\
\hline $80-89$ & $2,101(36.6)$ \\
\hline $90-$ & $455(7.9)$ \\
\hline Male sex & $3,326(57.9)$ \\
\hline Body weight (kg) & $55.7(47.2-65.1)$ \\
\hline Body mass index $\left(\mathrm{kg} / \mathrm{m}^{2}\right)$ & $22.7(20.2-25.5)$ \\
\hline Lean & $694(12.1)$ \\
\hline Normal & $3,381(58.9)$ \\
\hline Overweight & $1,319(23.0)$ \\
\hline Obese & $346(6.0)$ \\
\hline Hypertension & $3,627(63.2)$ \\
\hline Diabetes mellitus & $1,983(34.5)$ \\
\hline Chronic renal failure & $711(12.4)$ \\
\hline Chronic liver disease & $193(3.4)$ \\
\hline Chronic respiratory disease & $565(9.8)$ \\
\hline Cigarette smoking & $1,867(32.5)$ \\
\hline \multicolumn{2}{|l|}{ New York Heart Association } \\
\hline Class II & $1,873(32.6)$ \\
\hline Class III & $2,282(39.8)$ \\
\hline Class IV & $1,585(27.6)$ \\
\hline Barthel Index & $85(40-100)$ \\
\hline Barthel index $<60$ & $2,056(35.8)$ \\
\hline
\end{tabular}

and clinical data for approximately 8 million hospitalized patients per year from participating hospitals including all 82 academic hospitals in Japan. The main diagnosis, comorbidities on admission, and complications during hospitalization are recorded using the International Classification of Disease and Related Health Problems 10th Revision (ICD-10) codes. Chronic respiratory disease was defined as following ICD-10 codes; I27, J40-45, J47, J6067, and J70. Chronic liver disease was defined as following ICD-10 codes; B18, K70-74, and K76.

Ethics: This study was approved by the Institutional Review Board of the University of Tokyo [3501-(3)]. We conducted this study in accordance with the Declaration of Helsinki. Because of the anonymous nature of this database, the requirement for informed consent was waived.

Definition: We defined lean body weight, normal body weight, overweight, and obesity as body mass index < $18.5 \mathrm{~kg} / \mathrm{m}^{2}, 18.5-24.9 \mathrm{~kg} / \mathrm{m}^{2}, 25.0-29.9 \mathrm{~kg} / \mathrm{m}^{2}$ and $\geq 30$ $\mathrm{kg} / \mathrm{m}^{2}$, respectively. Low Barthel Index was defined as < $60 .{ }^{14)}$

Statistical analysis: Continuous and categorical data are presented as the median (interquartile range) and number (percentage), respectively. We evaluated the serial changes during 4 admissions in continuous data using the Jonckheere trend test, and categorical data using the CochranArmitage trend test. A probability value of $<0.05$ was considered to indicate a statistically significant difference. We performed statistical analysis using SPSS software (version 25, SPSS Inc., Chicago, IL, USA) and STATA (version 16, StataCorp LLC, College Station, TX, USA).

\section{Results}

We studied 466,921 patients aged $\geq 20$ years with NYHA class $\geq$ II, admitted and discharged between January 2010 and March 2018 with the main discharge diagnosis of HF defined by ICD-10 codes I50.0, I50.1, and I50.9. Exclusion criteria were as follows: 1) length of hospital stay $\leq 2$ days $(n=15,270)$ and 2$)$ major procedures under general anesthesia $(n=3,833)$. The final number of patients analyzed in this study was 447,818 . From among this total, we extracted 5,740 patients who were hospitalized 4 or more times.

Baseline clinical characteristics of the study population are summarized in Table I. The median age of the patients was $78(70-84)$ years, and 3,326 patients $(58 \%)$ were male. The median body mass index was 22.7 (20.225.5) $\mathrm{kg} / \mathrm{m}^{2}$. The incidences of hypertension, diabetes mellitus, and chronic renal failure were $63 \%, 35 \%$, and $12 \%$, respectively. Approximately $30 \%$ of the study population had New York Heart Association class IV symptoms. The median Barthel Index, which measures performance in activities of daily living, was 85 (40-100). Low Barthel Index was seen in 2,056 patients $(36 \%)$.

Serial changes in clinical presentations at hospital admission are shown in Table II. Body weight and body mass index decreased with each hospital admission. Patients with a higher NYHA class increased with each admission. Barthel Index decreased and the proportion of low Barthel Index increased with each admission.

The serial changes in clinical outcomes are summarized in Table III. The proportion of patients receiving respiratory support (J045 of the medical reimbursement code), hemodialysis, and inotropic agents increased with each admission, while that of intra-aortic balloon pumping decreased with an increase in the number of admissions. Compared to the second admission, the length of hospital stay was prolonged at the third and fourth admissions. Furthermore, the interval between hospitalizations was shortened with an increased number of admissions. Inhospital mortality was $8.8 \%$ at the fourth admission.

\section{Discussion}

It is well known that acute events due to worsened HF lead to further deterioration of cardiac function and progression of $\mathrm{HF}^{15)}$ However, there is a paucity of data on the association of repeated worsened HF with noncardiac clinical presentations and outcomes of patients with HF. We studied 5,740 patients requiring 4 or more hospital admissions owing to worsened HF using a nationwide inpatient database, and found that the presentations and outcomes of the patients serially declined with each hospital admission.

Body weight decreased by approximately $1 \mathrm{~kg}$ with each admission. Accordingly, lean patients increased and overweight and obese patients decreased. Rossignol, et al reported that body weight loss during follow-up was associated with higher mortality and adverse cardiovascular and non-cardiovascular outcomes. ${ }^{16)}$ Further, given that several studies have showed that obesity paradox could be applicable for $\mathrm{HF}^{17-20)}$ a decrease in body weight could be 
Table II. Serial Changes in Clinical Presentations

\begin{tabular}{|c|c|c|c|c|c|}
\hline & $\begin{array}{l}\text { 1st Admission } \\
\quad(n=5,740)\end{array}$ & $\begin{array}{l}\text { 2nd Admission } \\
\qquad(n=5,740)\end{array}$ & $\begin{array}{l}\text { 3rd Admission } \\
\quad(n=5,740)\end{array}$ & $\begin{array}{l}\text { 4th Admission } \\
\quad(n=5,740)\end{array}$ & $P$-value \\
\hline Body weight (kg) & $55.7(47.2-65.1)$ & $54.8(46.4-64.0)$ & $53.8(45.5-62.7)$ & $52.9(44.6-61.8)$ & $<0.001$ \\
\hline Body mass index $\left(\mathrm{kg} / \mathrm{m}^{2}\right)$ & $22.7(20.2-25.5)$ & $22.3(19.8-25.1)$ & $22.0(19.6-24.7)$ & $21.7(19.3-24.5)$ & $<0.0001$ \\
\hline Lean & $694(12.1)$ & $814(14.2)$ & $928(16.2)$ & $1,055(18.4)$ & \\
\hline Normal & $3,381(58.9)$ & $3,466(60.4)$ & $3,492(60.8)$ & $3,455(60.2)$ & \\
\hline Overweight & $1,319(23.0)$ & $1,152(20.1)$ & $1,047(18.2)$ & $978(17.0)$ & \\
\hline Obese & $346(6.0)$ & $308(5.4)$ & $273(4.8)$ & $252(4.4)$ & \\
\hline New York Heart Association & & & & & $<0.001$ \\
\hline Class II & $1,873(32.6)$ & $1,725(30.1)$ & $1,615(28.1)$ & $1,456(25.4)$ & \\
\hline Class III & $2,282(39.8)$ & $2,449(42.7)$ & $2,486(43.3)$ & $2,416(42.1)$ & \\
\hline Class IV & $1,585(27.6)$ & $1,566(27.3)$ & $1,639(28.6)$ & $1,868(32.5)$ & \\
\hline Barthel index & $85(40-100)$ & $85(40-100)$ & $75(40-100)$ & $65(30-100)$ & $<0.0001$ \\
\hline Feeding & $10(5-10)$ & $10(5-10)$ & $10(5-10)$ & $10(5-10)$ & \\
\hline Transfer & $15(10 / 15)$ & $15(10-15)$ & $10(10-15)$ & $10(5-15)$ & \\
\hline Grooming & $5(0-5)$ & $5(0-5)$ & $5(0-5)$ & $5(0-5)$ & \\
\hline Toilet use & $10(5-10)$ & $10(5-10)$ & $10(5-10)$ & $5(0-10)$ & \\
\hline Bathing & $5(0-5)$ & $5(0-5)$ & $0(0-5)$ & $0(0-5)$ & \\
\hline Mobility & $15(0-15)$ & $15(0-15)$ & $10(0-15)$ & $10(0-15)$ & \\
\hline Ascending/Descending stairs & $5(0-10)$ & $5(0-10)$ & $5(0-10)$ & $5(0-10)$ & \\
\hline Dressing & $10(5-10)$ & $10(5-10)$ & $5(5-10)$ & $5(0-10)$ & \\
\hline Bowel control & $10(5-10)$ & $10(5-10)$ & $10(5-10)$ & $10(5-10)$ & \\
\hline Bladder control & $10(5-10)$ & $10(5-10)$ & $10(5-10)$ & $10(5-10)$ & \\
\hline Barthel index $<60$ & $2,056(35.8)$ & $2,056(35.8)$ & $2,254(39.3)$ & $2,531(44.1)$ & $<0.001$ \\
\hline
\end{tabular}

Data are expressed as the median (interquartile range) or number (percentage).

Table III. Serial Changes in Clinical Outcomes

\begin{tabular}{|c|c|c|c|c|c|}
\hline & $\begin{array}{l}\text { 1st Admission } \\
(n=5,740)\end{array}$ & $\begin{array}{l}\text { 2nd Admission } \\
\qquad(n=5,740)\end{array}$ & $\begin{array}{l}\text { 3rd Admission } \\
\quad(n=5,740)\end{array}$ & $\begin{array}{c}\text { 4th Admission } \\
\quad(n=5,740)\end{array}$ & $P$-value \\
\hline Respiratory support & $755(13.2)$ & $760(13.2)$ & $813(14.2)$ & $976(17.0)$ & $<0.0001$ \\
\hline Intra-aortic balloon pumping & $32(0.6)$ & $12(0.2)$ & $12(0.2)$ & $13(0.2)$ & $<0.0001$ \\
\hline Extra-corporeal membrane oxygenation & $2(0.0)$ & $0(0.0)$ & $1(0.0)$ & $2(0.0)$ & 0.532 \\
\hline Inotropic use & $2,065(36.0)$ & $2,093(36.5)$ & $2,197(38.3)$ & $2,493(43.4)$ & $<0.0001$ \\
\hline Interval (days) & -------- & $120(39-320)$ & $85(33-226)$ & $80(30-207)$ & $<0.0001$ \\
\hline In-hospital death & -------- & -------- & -------- & $503(8.8)$ & -------- \\
\hline
\end{tabular}

Data are expressed as the median (interquartile range) or number (percentage).

recognized as a sign of a dismal prognosis. Malnutrition and cardiac cachexia, which are known as poor prognostic determinants of patients with $\mathrm{HF}^{21-23)}$ could coexist in patients with body weight loss. Further, Barthel Index decreased with each hospital admission and the proportion of low Barthel Index values increased from $35.8 \%$ at 1 st admission to $44.1 \%$ at 4 th admission, suggesting the deterioration of activities of daily living with each admission due to worsened HF. Activities of daily living are also associated with the outcomes of patients with HF. ${ }^{14,24)}$ These results might mirror the continuous deterioration of the non-cardiac general condition of HF patients with each hospital admission.

Regarding in-hospital outcomes, an increase in patients requiring advanced supports with each admission is thought to reflect that the proportions of patients developing life-threatening conditions increased with each admission. The proportion of patients undergoing hemodialysis increased with each admission. Although the detailed reason for hemodialysis could not be assessed in this study, worsened renal function during hospitalization has been associated with the adverse outcomes of patients with $\mathrm{HF}^{25-27)}$ Furthermore, patients requiring inotropic agents also increased from $36.0 \%$ at the 1 st admission to $43.4 \%$ at the 4th admission. Cardiogenic shock and low blood pressure were also reported to be associated with the adverse clinical outcomes of hospitalized HF patients. ${ }^{28,29)}$

In association with these various changes in clinical presentations and outcomes with each hospital admission, the length of hospital stay was prolonged and the interval of hospitalization was shortened with each hospital admission. As a consequence, in-hospital mortality was approximately $9 \%$ at the fourth admission, which was higher than that in a recent Japanese study. ${ }^{30)}$

This study has several clinical implications. Although repeated hospital admissions due to worsened $\mathrm{HF}$ are common in our daily clinical practice, detailed information on the serial changes in clinical presentations and outcomes of patients with repeated hospital admissions has been scarce. We believe that our results could clearly 


\section{5,740 patients requiring 4 or more times admissions to Worsend Heart Failure}

Median age of 78 years, 3,326 (58\%) Men

Jan 2010-March 2018

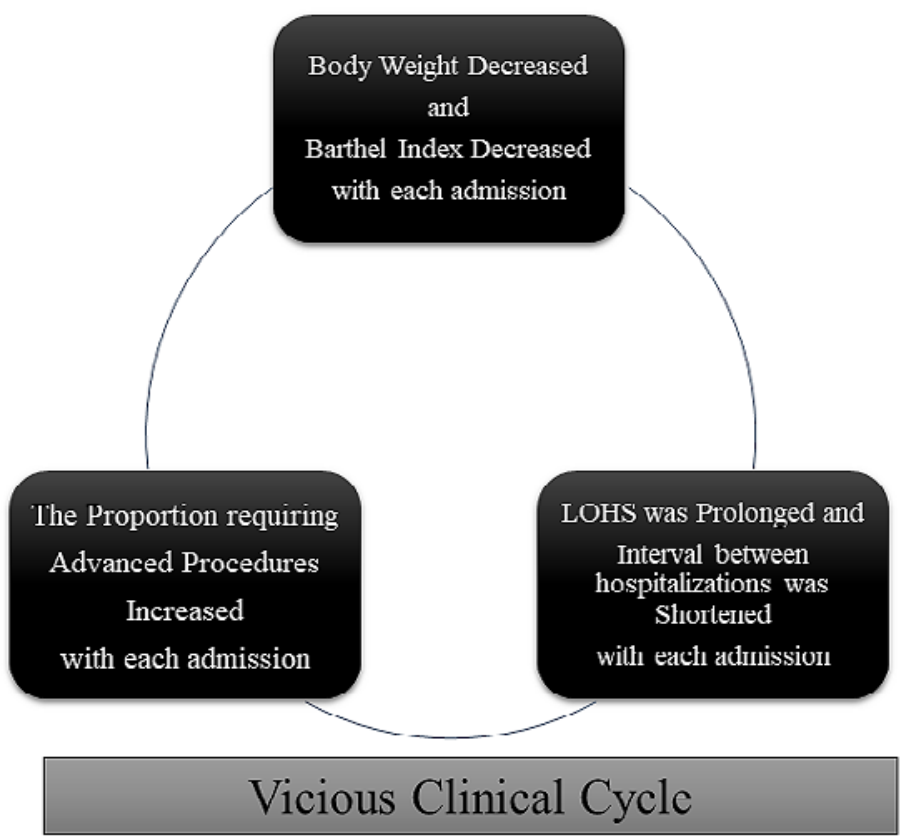

Figure. Summary of Study. Summarizes the main results of this study. LOHS indicates length of hospital stay.

demonstrate how the clinical presentations of the patients declined with each hospital admission and led to adverse outcomes (Figure). Furthermore, it is also important to share these concrete data with healthcare professionals, patients and their families, and to reconfirm the significance of prevention of HF readmission.

There are several limitations to this study. Although the validity of the diagnoses and procedures in our database was reported to be high, ${ }^{31)}$ recorded diagnoses are generally considered less well validated because of the nature of administrative data and retrospective studies. Our database lacked information on several factors that were potentially associated with the outcomes, including blood pressure, HF etiology, and left ventricular ejection fraction. Therefore, the applicability of our findings on which types of heart failure such as HF with reduced ejection fraction, HF with preserved ejection fraction, ischemic cardiomyopathy, and non-ischemic cardiomyopathy remains unclear. Generally, body weight and the Barthel Index decrease with age, so we were unable to separate the impact of physiological aging and repeated hospital admissions on the changes in body weight and Barthel Index. Further investigations are required to clarify the determinants of changes in these clinical manifestations of patients with HF.

\section{Conclusion}

This is the first large-scale real-world study on the serial changes in clinical characteristics and outcomes of HF patients requiring repeated hospital admissions due to worsened HF, suggesting that repeated hospital admissions might adversely affect not only cardiac function but also the non-cardiac general status of patients with $\mathrm{HF}$ and lead to a vicious clinical cycle.

\section{Disclosure}

Research funding and scholarship funds were obtained by Hidehiro Kaneko and Katsuhito Fujiu from Medtronic Japan, Abbott Medical Japan, Boston Scientific Japan, and Fukuda Denshi, Central Tokyo.

Conflicts of interest: None to declare.

\section{References}

1. Go AS, Mozaffarian D, Roger VL, et al. Heart disease and stroke statistics--2014 update: a report from the American Heart Association. Circulation 2014; 129: e28-292.

2. Gheorghiade M, Vaduganathan M, Fonarow GC, Bonow RO Rehospitalization for heart failure: problems and perspectives. J Am Coll Cardiol 2013; 61: 391-403.

3. Ponikowski P, Voors AA, Anker SD, et al. 2016 ESC Guidelines for the diagnosis and treatment of acute and chronic heart failure: The Task Force for the diagnosis and treatment of acute and chronic heart failure of the European Society of Cardiology (ESC). Developed with the special contribution of the Heart Failure Association (HFA) of the ESC. Eur J Heart Fail 2016; 18: 891-975.

4. Hiki M, Iwata H, Takasu K, et al. Elevated heart rate in combination with elevated blood pressure predicts lower cardiovascular mortality in acute decompensated heart failure. Int Heart $\mathbf{J}$ 2020; 61: 308-15. 
5. Abe S, Yoshihisa A, Ichijo Y, et al. Recovered left ventricular ejection fraction and its prognostic impacts in hospitalized heart failure patients with reduced ejection fraction. Int Heart J 2020; 61: 281-8.

6. Kaneko H, Suzuki S, Goto M, et al. Incidence and predictors of rehospitalization of acute heart failure patients. Int Heart J 2015; 56: 219-25

7. Pocock SJ, Wang D, Pfeffer MA, et al. Predictors of mortality and morbidity in patients with chronic heart failure. Eur Heart J 2006; 27: 65-75.

8. Solomon SD, Dobson J, Pocock S, et al. Influence of nonfatal hospitalization for heart failure on subsequent mortality in patients with chronic heart failure. Circulation 2007; 116: 1482-7.

9. Setoguchi S, Stevenson LW, Schneeweiss S. Repeated hospitalizations predict mortality in the community population with heart failure. Am Heart J 2007; 154: 260-6.

10. Kaneko H, Itoh $\mathrm{H}$, Yotsumoto $\mathrm{H}$, et al. Association between the number of hospital admissions and in-hospital outcomes in patients with heart failure. Hypertens Res 2020 (in press).

11. Yagi M, Yasunaga $\mathrm{H}$, Matsui $\mathrm{H}$, et al. Impact of rehabilitation on outcomes in patients with ischemic stroke: A Nationwide Retrospective Cohort Study in Japan. Stroke 2017; 48: 740-6.

12. Shigemi D, Isogai S, Uda K, et al. Association between rehabilitation during hospitalization and perinatal outcomes among pregnant women with threatened preterm birth. J Matern Fetal Neonatal Med 2019; 1-6.

13. Kaneko H, Itoh $\mathrm{H}$, Yotsumoto $\mathrm{H}$, et al. Characteristics and outcomes of super-elderly patients (Aged $>=90$ years) hospitalized for heart failure - Analysis of a Nationwide Inpatient Database-. Circ Rep 2020; 2: 393-9.

14. Uemura Y, Shibata R, Takemoto K, et al. Prognostic Impact of the preservation of activities of daily living on post-discharge outcomes in patients with acute heart failure. Circ J 2018; 82: 2793-9.

15. Gheorghiade M, De Luca L, Fonarow GC, Filippatos G, Metra M, Francis GS. Pathophysiologic targets in the early phase of acute heart failure syndromes. Am J Cardiol 2005; 96: 11G-7G.

16. Rossignol P, Masson S, Barlera S, et al. Loss in body weight is an independent prognostic factor for mortality in chronic heart failure: insights from the GISSI-HF and Val-HeFT trials. Eur J Heart Fail 2015; 17: 424-33.

17. Horwich TB, Fonarow GC, Hamilton MA, MacLellan WR, Woo MA, Tillisch JH. The relationship between obesity and mortality in patients with heart failure. J Am Coll Cardiol 2001; 38: 78995.

18. Lavie CJ, Osman AF, Milani RV, Mehra MR. Body composition and prognosis in chronic systolic heart failure: the obesity para- dox. Am J Cardiol 2003; 91: 891-4.

19. Curtis JP, Selter JG, Wang Y, et al. The obesity paradox: body mass index and outcomes in patients with heart failure. Arch Intern Med 2005; 165: 55-61.

20. Lavie CJ, Alpert MA, Arena R, Mehra MR, Milani RV, Ventura HO. Impact of obesity and the obesity paradox on prevalence and prognosis in heart failure. JACC Heart Fail 2013; 1: 93102 .

21. Kaneko H, Suzuki S, Goto M, et al. Geriatric nutritional risk index in hospitalized heart failure patients. Int J Cardiol 2015; 181: $213-5$.

22. Anker SD, Coats AJ. Cardiac cachexia: a syndrome with impaired survival and immune and neuroendocrine activation. Chest 1999; 115: 836-47.

23. Mustafa I, Leverve X. Metabolic and nutritional disorders in cardiac cachexia. Nutrition 2001; 17: 756-60.

24. Dunlay SM, Manemann SM, Chamberlain AM, et al. Activities of daily living and outcomes in heart failure. Circ Heart Fail 2015; 8: 261-7.

25. Herout PM, Harshaw Q, Phatak H, et al. Impact of worsening renal function during hospital admission on resource utilization in patients with heart failure. Am J Cardiol 2010; 106: 1139-45.

26. Maeder MT, Rickli H, Pfisterer ME, et al. Incidence, clinical predictors, and prognostic impact of worsening renal function in elderly patients with chronic heart failure on intensive medical therapy. Am Heart J 2012; 163: 407-14.

27. Damman K, Valente MA, Voors AA, O'Connor CM, van Veldhuisen DJ, Hillege HL. Renal impairment, worsening renal function, and outcome in patients with heart failure: an updated meta-analysis. Eur Heart J 2014; 35: 455-69.

28. Fonarow GC, Adams KF Jr, Abraham WT, et al. Risk stratification for in-hospital mortality in acutely decompensated heart failure: classification and regression tree analysis. JAMA 2005; 293: 572-80.

29. Patel PA, Heizer G, O'Connor CM, et al. Hypotension during hospitalization for acute heart failure is independently associated with 30-day mortality: findings from ASCEND-HF. Circ Heart Fail 2014; 7: 918-25.

30. Shiraishi Y, Kohsaka S, Sato N, et al. 9-year trend in the management of acute heart failure in Japan: A report from the National Consortium of Acute Heart Failure Registries. J Am Heart Assoc 2018; 7: e008687.

31. Yamana H, Moriwaki M, Horiguchi H, Kodan M, Fushimi K, Yasunaga H. Validity of diagnoses, procedures, and laboratory data in Japanese administrative data. J Epidemiol 2017; 27: 476-82. 